\section{Review: Individual behavioural therapy reduces symptoms of depression}

\section{QUESTION}

Question: How effective are behavioural therapies for depression?

Outcomes: Depressive symptoms (either self-rated—eg, Beck Depression Inventory, or clinician rated-eg, Hamilton Depression Rating Scale).

\section{METHODS}

Design: Systematic review with meta-analysis.

Data sources: MEDLINE, EMBASE, PsycINFO, Cochrane Library, DARE, CINAHL, AMED and the British Nursing Index were searched from inception to January 2006 for randomised controlled trials (RCTs). Reference lists of identified studies were hand searched.

Study selection and analysis: Two reviewers appraised studies and selected RCTs of behavioural therapy in adults (aged $\geqslant 16$ years) with a primary diagnosis of depression. Treatments could be delivered in a community or inpatient setting. Behavioural therapy had to be individual, time limited, and based on changing the behavioural consequences of environmental or cognitive triggers. Comparator treatments could include cognitive behavioural therapy (CBT) or cognitive therapy, brief psychotherapies, supportive counselling, treatment as usual, waiting list or other inactive control. Two reviewers rated study quality and extracted data. Authors were contacted to obtain missing data, but if this was not successful, standard deviations were imputed from other related studies. Random effect metaanalyses were conducted using RevMan software. Separate meta-analyses were carried out for different comparator groups. Heterogeneity was investigated using the $\mathrm{I}^{2}$ statistic.

\section{MAIN RESULTS}

Twenty RCTs were identified which met inclusion criteria, but 3 of the RCTs did not have enough data to be included in the meta-analyses. Behavioural therapy reduced depressive symptoms compared to control at the end of treatment (12 RCTs, 459 participants; controls included waiting lists, treatment as usual and relaxation). There was no significant difference between behavioural therapy and cognitive therapy or CBT in depressive symptoms post-treatment or at an average of 4 months' follow-up (post-treatment: 12 RCTs, 476 participants; follow up: 8 RCTs, 271 participants). Behavioural therapy reduced depressive symptoms posttreatment and at an average of 4.5 months' follow-up compared to brief psychotherapy (post-treatment: 3 RCTs, 166 participants; follow up: 2 RCTs, 96 participants). Compared to supportive counselling, behavioural therapy reduced symptoms of depression post-treatment (2 RCTs, 45 participants). See online table.

\section{CONCLUSIONS}

Individual behavioural therapy reduces symptoms of depression more than control, supportive counselling or brief psychotherapy. It provides similar reductions in depressive symptoms to cognitive therapy and CBT.

\section{ABSTRACTED FROM}

Ekers D, Richards D, Gilbody S. A meta-analysis of randomized trials of behavioural treatment of depression. Psychol Med 2008;38:611-23.

Correspondence to: D Ekers, Tees Esk \& Wear Valleys NHS Trust/University of York Department of Health Sciences, The Health Centre, Newcastle Road, Chester le Street, County Durham, UK; David.Ekers@cddps.nhs.uk

Source of funding: Unclear.

- Additional notes and a table are published online only at http://ebmh.bmi.com/ content/vol11/issue4 ognitive behaviour therapy (CBT) is the most well-researched and established psychotherapeutic approach for treating depression. ${ }^{1}$ However, as with antidepressant medications, ${ }^{2}$ many unanswered questions remain about the mechanisms through which CBT for depression produces its therapeutic effects. Is it the distinctive cognitive techniques that are producing the majority of the benefits, or are the other non-cognitive elements also a key part of the treatment?

The meta-analysis by Ekers and colleagues provides a tantalising piece of the puzzle. They conducted a comprehensive quantitative review of 17 clinical trials of behaviour therapy (BT) for depression, which did not include specific cognitive therapy techniques. The authors reasoned that if BT produces the same effects as the more comprehensive CBT package, then this would suggest that the cognitive elements are unnecessary for achieving therapeutic gains. As CBT is a more complicated treatment to train therapists to implement, BT would be an attractive alternative given its apparent parsimony. The results of the meta-analysis appeared to confirm this premise, with BT being equally as effective as $\mathrm{CBT}$, and more effective than control interventions, including treatment as usual, brief psychodynamic therapy and supportive therapy. The dropout rates between BT and the other treatments were similar, and BT appeared to work well regardless of initial severity, length of treatment, or level of therapist training. The authors did not specifically test the comparability of BT and antidepressant medications.

Another meta-analysis reached similar conclusions about the equivalence of $\mathrm{CBT}$ and $\mathrm{BT}^{1}$ and a recent large-scale clinical trial of BT showed that it was as effective as antidepressant medication for depression. ${ }^{3}$ While BT and CBT were equally as effective for lower-levels of depression severity, patients with severe depression specifically benefited more from $\mathrm{BT}$ than $\mathrm{CBT} .{ }^{3}$ Behavioural activation, which involves graded activity and goal scheduling, is the central component of BT for depression and may be related to a more general mechanism of change that is operating in many different treatments for depression, especially CBT. The UK's National Health Service recently allocated $£ 173$ million to train more
CBT therapists for treating depression and anxiety. Ekers and colleagues' meta-analysis suggests that the money may be best spent training better behaviour therapists.

\section{Brandon A Gaudiano, PhD}

Alpert Medical School of Brown University and Butler Hospital, Providence, Rhode Island, USA

\section{Competing interests: None.}

1. Butler AC, Chapman JE, Forman EM, et al. The empirical status of cognitive-behavioral therapy: a review of meta-analyses. Clin Psychol Rev 2006;26: $17-31$.

2. Kirsch I, Deacon BJ, Huedo-Medina TB, et al. Initia severity and antidepressant benefits: a meta-analysis of data submitted to the Food and Drug Administration. PLOS Med 2008:5:e45.

3. Dimidjian S, Hollon SD, Dobson KS, et al. Randomized trial of behavioral activation, cognitive therapy, and antidepressant medication in the acute treatment of adults with major depression. $J$ Consult Clin Psychol 2006:74:658-70.

4. Talk therapy plans under fire. BBC News [Internet] 6 July 2008. Available at http://news.bbc.co.uk/go/pr/ fr/-/2/hi/health/7486132.stm (accessed 6 July 2008). 